\title{
Sala de espera: um suporte ao atendimento odontológico \& à saúde bucal
}

\author{
| Waiting room: a support to dental and oral health care service
}

\author{
Beatriz Lemos Stutz ${ }^{1}$ \\ Ludmila Cavalceanti de Mendonța ${ }^{2}$ \\ Samara dos Santos Rodrigues fomes ${ }^{3}$
}

\begin{abstract}
Resumo
A presente pesquisa é resultado do processo de investigação sobre as influências de atividades desenvolvidas por docentes e estudantes da Escola Técnica de Saúde na sala de espera do Hospital Odontológico da Universidade Federal de Uberlândia, junto aos pacientes da comunidade local, bem como seus hábitos de higiene bucal e de seus acompanhantes. Por meio de um questionário com questões abertas, respondido pelos acompanhantes de menores de dezoito anos, investigaram-se também as expectativas e o nível de satisfação em relação ao atendimento realizado. Os resultados obtidos demonstram a importância do desenvolvimento desse tipo de projeto de extensão na promoção da saúde e do bem-estar, não somente dos pacientes, como também daqueles que os acompanham ao atendimento, aproximando-os da equipe de trabalho, mediante ações lúdicas $e$ educativas.
\end{abstract}

\section{Abstraet}

This research is a result of an investigating of process about influences on activities developed by teachers and students from the Technical School of Health in the waiting room of the Dental Hospital of the Federal University of Uberlandia, with patients from the local community as well as their mouth hygiene habits and their companions. Through a questionnaire with open questions answered by companions of children under eighteen yeas old, we also investigated the expectations and satisfaction level with the provided services. The results demonstrate the importance of developing this type of extension project in promoting health and welfare not only of patients but also with those who come to with them, through recreational and educational activities.
Deseritores: educaţão profissional; saide eoletiva; sala de espera; saiude bucal

Keywords:professional education, public health, waiting room, oral health.
'Doutora em Educação pela Universidade Federal de Uberlândia, docente da Eseola Téeniea de Saíde da Universidade Federal de Uberlândia [ESTES/UFU]..

\footnotetext{
${ }^{2}$ Mestre em Reabilitaķão Oral pela Universidade Federal de Uberlândia, docente da Escola Téeniea de Saúde da Universidade Federal de Uberlândia [ESTES/UFU].

${ }^{3}$ Doutora em Ciêneias da Saúde pela Universidade de Brasilia, docente da Eseola Téeniea de Saúde da Universidade Federal de Uberlândia [ESTES/UFU].
}

Para correspondência:

Samara dos Santos Rodrigues Gomes

E-mail:samaraunb@yahoo.com.br 
Introdução

O projeto Sala de Espera em Odontologia foi criado no ano de 2002 e vem sendo desenvolvido até os dias atuais, como parte do trabalho para formação de profissionais técnicos de nível médio da Escola Técnica de Saúde da Universidade Federal de Uberlândia (ESTES/UFU). Desde então, a operacionalização desse projeto ocorre por meio da realização de atividades lúdicas e educacionais na sala de espera do Hospital Odontológico da Faculdade de Odontologia/UFU, complementares às práticas supervisionadas que compõem a matriz curricular do programa de ensino desenvolvido com alunos do Curso Técnico em Saúde Bucal. Tais atividades acontecem semanalmente e estão voltadas para os usuários do sistema de saúde na faixa etária de 5 a 35 anos, residentes no município de Uberlândia.

Caracterizada como projeto de ensino, extensão e pesquisa, esta investigação envolveu um total de 450 usuáriosde 5 a 35 anos, entre pacientes e acompanhantes, atendidos Hospital Odontológico da Faculdade de Odontologia/UFU, e foi aprovada pelo Comitê de Ética em Pesquisa com Seres Humanos da Universidade Federal de Uberlândia - MG, sob o protocolo $\mathrm{n}^{\circ}$. 077/10.

Tais atividades têm o intuito de proporcionar aos estudantes, que delas participam, instrumentos teórico-práticos referentes à compreensão dos aspectos emocionais e comportamentais dos pacientes envolvidos no atendimento odontológico e uma ampla formação, para além das atividades técnicas específicas da área, circunscritas no âmbito da cidadania.

Considerando que ao aguardar o atendimento, a clientela, em sua maioria, fica ociosa, propiciando em muitos casos o desenvolvimento de ansiedades, busca-se contribuir para diminuição deste aspecto em relação aos procedimentos odontológicos e para melhoria das condições de saúde e higiene bucal dos pacientes por meio de processos educativos.

Esse trabalho vai ao encontro da necessidade apontada por pesquisadores da saúde, entre eles Paixão e Castro ${ }^{1}$, de união de esforços entre acadêmicos e profissionais dessa área em torno de projetos de caráter interdisciplinar, de cunho educativo e preventivo, direcionados aos usuários da rede de assistência existente. Essas autoras, ao relatarem sua experiência em um grupo de sala de espera, a partir da qual foram desenvolvidas sistematicamente ações socioeducativas a usuários do Sistema Único de Saúde (SUS), chamam a atenção para a importância desse tipo de trabalho enquanto multiplicador de uma política de saúde voltada para a qualidade de vida das pessoas e, ao mesmo tempo, para o fato de as universidades ampliarem os espaços a eles reservados, estimulando a compreensão da realidade e necessidades existentes no setor de saúde pública.

Para Silva ${ }^{2}$ a educação é uma importante estratégia para a promoção da saúde, uma vez que possibilita às pessoas desenvolver a capacidade de intervenção sobre suas vidas e a sala de espera é um espaço popular, no qual a atividade multidisciplinar motiva reflexões e favorece a produção coletiva do saber. Assim, este trabalho vem ao encontro dessa necessidade apontada, de ampliação de ações socioeducacionais efetivas no setor da saúde pública (Foto 1) bem como, produção de saberes a ele relacionados.

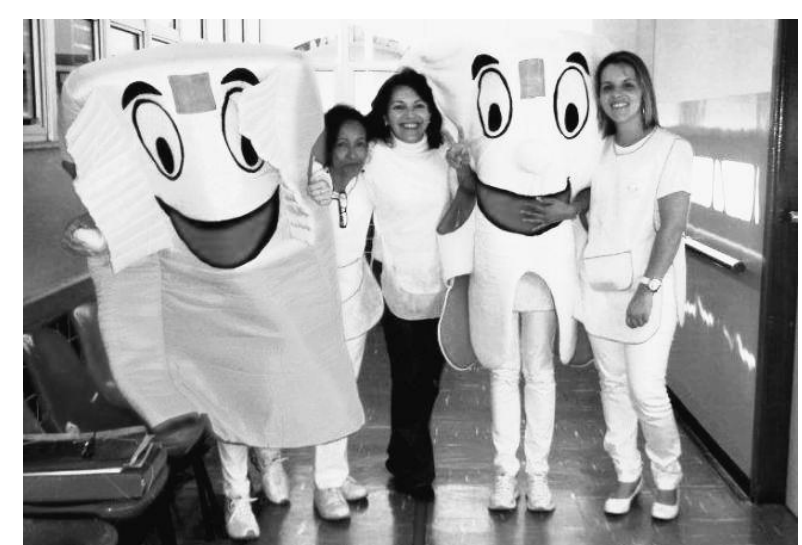

Foto 1. Membros da equipe participante do Projeto Sala de Espera em Odontologia ano 2012 (ESTES/UFU) realizando atividade em saúde bucal no Hospital Odontológico da FOUFU/UFU.

Resultado de um esforço conjunto entre docentes, discentes e técnicos administrativos da ESTES/UFU, tem-se verificado, ao longo desse período, que a sala de espera da clínica, aqui referida, constitui-se em um espaço rico não apenas para a formação dos alunos, como também para uma aproximação entre eles, os profissionais e a comunidade atendida. Além disso, facilita a construção de um trabalho educativo voltado para a manutenção da saúde bucal e prevenção de doenças periodontais.

$\mathrm{O}$ estudo aqui apresentado é resultado de 
pesquisa realizada mediante a aplicação de questionário dirigido aos pais ou responsáveis por crianças atendidas nos anos de 2010 e 2011.

\section{Objetivos}

As ações realizadas dentro do projeto Sala de Espera em Odontologia têm como objetivos mobilizar e despertar a clientela atendida na clínica do Hospital Odontológico/FOUFU pela equipe de professores e alunos do Curso Técnico em Saúde bucal da ESTES/UFU, quanto aos cuidados para uma saúde bucal adequada, assim como proporcionar a estes últimos aulas práticas complementares, relacionadas a comportamento humano, educação em saúde e atendimento ao público. Visam, ainda, desenvolver um trabalho educativo na área de saúde bucal junto aos pacientes e acompanhantes, com técnicas corretas de escovação e cuidados gerais para prevenção de cáries.

De forma continuada, objetiva desenvolver atividades com pacientes, direcionadas para a diminuição da ansiedade na sala de espera, tais como: desenhos, recortes, colagens, construção coletiva de histórias, dobraduras, massa de modelar, confecção de adereços e enfeites pessoais, cartazes, cartões, jogos e atividades de relaxamento.

Nesse sentido, busca desenvolver no aluno a capacidade de observação e análise do comportamento, aspectos emocionais dos pacientes envolvidos no atendimento odontológico, assim como habilidades quanto ao trabalho em equipe e relacionamento interpessoal, orientando pacientes e acompanhantes quanto aos serviços prestados na clínica.

\section{Metodologia}

A presente investigação foi aprovada pelo Comitê de Ética em Pesquisa da Universidade Federal de Uberlândia em 21 de maio de 2010, sob o Protocolo de Registro $n^{\circ}$. 077/10, de acordo com as atribuições definidas na Resolução CNS 196/96. Consiste na análise qualitativa de atividades educativas e lúdicas na área de saúde bucal, realizadas semanalmente por alunas do Curso Técnico em Saúde Bucal da ESTES/UFU na sala de espera da Clínica do Hospital Odontológico da Faculdade de Odontologia da UFU, sob a orientação da equipe de professoras envolvidas com esse projeto.

$\mathrm{O}$ instrumento de pesquisa utilizado consistiu em um questionário, aplicado aos acompanhantes da clientela atendida na faixa etária de 5 a 13 anos. Excluíram-se da pesquisa acompanhantes que não fossem os pais ou os responsáveis diretos pelos pacientes, já que muitos deles são levados ao atendimento por parentes ou amigos, que não têm conhecimento sobre seus hábitos cotidianos.

O preenchimento do questionário ocorreu a partir do segundo dia de atendimento odontológico de cada paciente, uma vez que algumas questões estão relacionadas a orientações dadas pelos profissionais aos acompanhantes e à verificação do nível de satisfação deles com os serviços prestados. A amostragem foi definida tendo-se como critério a saturação das respostas e considerando ainda a dificuldade de muitos acompanhantes em responderem ao questionário, necessitando para isto do acompanhamento direto da docente responsável e / ou de alunos do curso de Técnico em Saúde Bucal durante seu preenchimento.

Foram analisados 11 questionários em 2010, referentes a crianças na faixa etária entre 06 a 10 anos e 18 em 2011, com idade entre 05 a 13 anos. Todas elas estavam cursando o ensino fundamental, sendo que no primeiro ano pesquisado, 04 eram do sexo feminino e 07 do sexo masculino e, no segundo ano, o4 eram do sexo feminino e 14 do sexo masculino. Quanto aos acompanhantes, a maioria era composta por mães dos pacientes e os demais eram pais, avós, irmãs e tias, com grau de escolaridade compreendido entre o ensino fundamental incompleto e superior completo (tabela 1).

\begin{tabular}{lllll}
\hline Ano & Parentesco & & Escolaridade & \\
\hline 2010 & Mãe & 07 & Fundamental incompleto & 02 \\
& Pai & 01 & Fundamental completo & 04 \\
& Irmã & 01 & Médio incompleto & 02 \\
& Avó & 01 & Médio completo & 02 \\
& Tia & 01 & Superior incompleto & 01 \\
Total & & 11 & & 11 \\
\hline 2011 & Mãe & 13 & Fundamental incompleto & 06 \\
& Pai & 03 & Fundamental completo & 02 \\
& Madrasta & 01 & Médio incompleto & 01 \\
& Tio & 01 & Médio completo & 08 \\
& & & Superior completo & 01 \\
Total & & 18 & & 18 \\
\hline
\end{tabular}

Tabela 1. Dados dos acompanhantes

Fonte: pesquisa direta 
As questões que compõem esse questionário abordam aspectos referentes a tratamentos realizados anteriormente, expectativas em relação ao tratamento odontológico atual, realizado na clínica, verificação da existência ou não de orientações dadas aos acompanhantes do paciente logo após o primeiro atendimento, nível de satisfação em relação a ele, tempo de espera entre a triagem e a marcação de horário para o atendimento, sentimento do acompanhante enquanto aguarda na sala de espera, seu interesse quanto à obtenção de informações sobre Saúde e Higiene Bucal e, por fim, quais são os hábitos de higiene bucal da clientela atendida e seus acompanhantes.

Para verificação dos aspectos acima citados, foram incluídas, no questionário, questões dentre as quais podem ser destacadas: Seu filho já fez algum tratamento dentário antes? Como considera a experiência de seu filho em relação ao tratamento realizado? Você tem recebido orientação sobre o tratamento realizado em seu filho nesta clínica? O que você sabe sobre o trabalho que é realizado? Você gostaria de obter informações sobre Saúde e Higiene Bucal na sala de espera?

Quanto à verificação de hábitos em higiene bucal, optou-se pelas seguintes questões abertas: O que você utiliza ao realizar sua higiene bucal? Quantas vezes ao dia você a realiza? O que seu filho utiliza ao realizar sua higiene bucal? Quantas vezes ao dia a realiza?

Vale ressaltar que a opção por questões abertas, como estratégia de investigação dos hábitos em higiene bucal, ocorreu após a fase piloto para construção do instrumento de pesquisa, quando se pôde constatar que o preenchimento de questões no modelo múltipla escolha estava induzindo a respostas pelo maior número de escovações e ações referentes a ela. Isto não significa que questões abertas tenham sido garantia de respostas sinceras e que correspondam de fato à realidade dos hábitos adotados pelos sujeitos. Contudo, percebeu-se que ao serem incluídas opções com número de vezes em que é feita a higiene bucal e quais recursos são utilizados, as pessoas tendiam a marcar o maior número existente e também a utilização de fio dental e enxaguante bucal, talvez por considerá-las como sendo as respostas esperadas.

A aplicação deste questionário vai ao encontro da importância apontada por pesquisadores na área, entre eles Pauleto $^{3}$, de programas odontológicos que levantem e interpretem as necessidades da população de menor acesso aos serviços de saúde em odontologia. Assim, informações sobre o nível de satisfação da clientela em relação ao atendimento odontológico, conhecimentos e dúvidas que possuem na área de saúde bucal, expectativas em relação ao tratamento oferecido e impacto das atividades realizadas na sala de espera sobre $o$ comportamento e higiene bucal das crianças e adolescentes atendidos, tornam-se instrumentos extremamente úteis na construção de um atendimento de qualidade à população, como também dão suporte à elaboração de estratégias de intervenção que possibilitem desenvolvimento ou mudança de hábitos dos usuários e acompanhantesem relação a sua higiene e saúde bucal.

\section{Resultados e diseussão}

Para Possobon ${ }^{4}$, a situação de tratamento odontológico é geradora de ansiedade não apenas para o paciente como também para a equipe que a executa. Nesse aspecto, requer habilidades dos profissionais que dela participam no sentido de possuir um repertório de estratégias diferenciadas de manejo do comportamento, que possam tornar sua rotina de trabalho menos estressante, promovendo ao mesmo tempo maior conforto e segurança aos usuários durante a realização dos procedimentos.

Cardoso e Loureiro $^{5}$ chamam atenção para a importância dos cuidados com os aspectos emocionais e afetivos das crianças durante o atendimento odontológico e que, programas de saúde bucal voltados para a comunidade devem levar em conta tais fatores, sem o que, podem tornar-se ineficazes.

No contexto da clínica-escola, as manifestações de estresse por parte da clientela atendida dificultam comportamentos de colaboração para com as práticas odontológicas realizadas. Dessa forma, é premente a necessidade de se criarem condições para que o início dos trabalhos clínicos favoreça o desenvolvimento de habilidades para lidar com tais manifestações, o envolvimento de equipes multidisciplinares e 
uma real aproximação entre os profissionais e usuários dos serviços prestados, com vistas a uma maior integralidade das ações de saúde, mediante a criação de espaços para esse fim ${ }^{6}$.

A partir de tais considerações, o projeto Sala de Espera em Odontologia, além de importante estratégia para enfrentamento do medo e da ansiedade que envolvem o atendimento clínico e os usuários do sistema de saúde, por meio de atividades lúdico-educacionais, proporciona também aos alunos do Curso Técnico em Saúde Bucal da ESTES/UFU situações de aprendizagem em integração com os profissionais nele envolvidos, sendo espaço de crescimento humano e formação profissional.

Por meio desse projeto, tem-se buscado proporcionar ampla formação aos alunos para além das habilidades técnicas específicas da área, contribuindo para sua formação enquanto cidadão, além de estimular o desenvolvimento de habilidades para lidar com aspectos emocionais envolvidos no atendimento ao público, nos cuidados e na prevenção em higiene bucal.

Tendo como princípio educativo a aproximação dos alunos e profissionais a ele ligados com a comunidade que utiliza esse serviço, incluindo-se os acompanhantes, objetivase estimular o desenvolvimento de instrumentos de pesquisa, que permitam uma leitura de aspectos econômicos, sociais e culturais presentes no seu cotidiano, fornecendo subsídios para a compreensão de fatores que concorrem para a criação de hábitos e valores presentes na forma como as crianças e adolescentes atendidos agem com relação aos cuidados com o próprio corpo e sua saúde bucal.

Tomita ${ }^{7}$, ao realizarem estudos sobre saúde bucal, enfatizam a importância de métodos educativos dinâmicos e participativos sobre a melhoria dos padrões de higiene bucal da clientela atendida, sendo fundamental na mudança de hábitos independente de sua inserção social.

Marra $^{8}$, ao analisarem a inclusão da odontologia nos planos municipais de saúde das cidades integrantes da gerência regional de saúde de Uberlândia-MG, chamam a atenção para o fato de que é comum a repetição de modelos de atenção à saúde bucal superados ou inadequados à sua realidade, não desenvolvendo atividades em educação nessa área, tão imprescindíveis para mudanças de hábitos da população que resultem em ações preventivas.
A análise dos dados, na pesquisa aqui apresentada, mostra que a maioria dos entrevistados, ao levar os filhos para atendimento odontológico, espera por um tratamento de qualidade, com bons resultados, correspondendo a $63,63 \%$ em 2010 e $61,11 \%$ em 2011. Os demais, elencaram como expectativas, em relação ao tratamento, que os filhos sejam orientados quanto aos cuidados gerais com a higiene bucal e prevenção das cáries, contribuindo para uma dentição saudável $(36,36 \%$ em 2010 e 38,88\% em 2011).

A resposta de uma das mães, ao falar sobre suas expectativas, ilustra a importância do relacionamento profissional-paciente: Espero um bom tratamento, de qualidade. Meu filho gosta de vir a esta clínica por causa do atendimento das pessoas. Quando a pessoa é tratada com carinho ela quer voltar. Quando isso não acontece, ela não volta.

Outra fala chama atenção para a qualidade do atendimento no setor público: Não é porque é público que não pode ter um bom tratamento.

Constata-se que, um percentual significativo dos pacientes possuía uma história de atendimentos anteriores, correspondente a $36,36 \%$ em 2010 e 77,77\% em 2011 (tabela 2).

Ao serem questionados se haviam recebido orientações sobre o atendimento realizado nas crianças, percebe-se uma acentuada diferença entre as respostas dos acompanhantes nos anos pesquisados, tendo sido apontado maior percentual de respostas positivas em 2011, como se pode observar nos dados a seguir (tabela 2).

\begin{tabular}{lcc}
\hline ANO & 2010 & 2011 \\
\hline Sim & 06 & 14 \\
Não & 05 & 03 \\
Sem resposta & 00 & 01 \\
Total & 11 & 18
\end{tabular}

Tabela 2. Frequência de orientações recebidas após o atendimento.

Fonte: pesquisa direta

Esse fato pode ser explicado pela constatação, durante as avaliações feitas pelo corpo docente responsável pela execução do projeto Sala de Espera, da necessidade em dar retorno mais detalhado aos acompanhantes sobre o tratamento 
realizado nas crianças, ao perceber-se que um número significativo demonstrava interesse em obtê-lo. Observou-se, ao longo deste trabalho que, embora as alunas do curso de TSB fossem orientadas a levar a criança até seu acompanhante após o atendimento, fazendo algumas observações sobre o que havia sido realizado, muitas vezes eram informações superficiais que não esclareciam, inclusive, sobre as etapas subsequentes até o término do tratamento.

Percebeu-se, ao longo de todo o processo, que esse fator resultou em aumento do nível de satisfação da clientela, tendo sido apontado como um aspecto que reforça a importância dessas informações, não apenas para tranquilidade dos pais ou responsáveis, mas fundamentalmente pela influência que o conhecimento pode acarretar na melhoria das condições de higiene e saúde bucal das crianças, como demonstram as falas de participantes da pesquisa. O primeiro diz: porque eu como pai quero acompanhar todos os passos do tratamento e poder orientar para ele ter uma melhor saúde bucal. O segundo afirma: as orientações são importantes para seu próprio bem-estar em casa e meu filho acaba aprendendo mais. A terceira declarou: porque através das informações fico mais atenta ao tratamento dela e como fazer.

Ao serem questionados sobre seus sentimentos na sala de espera enquanto aguardava pelo atendimento da criança, a maioria afirmou ficar tranquila, sendo que em 2010 apenas uma mãe frisou sentir-se ansiosa por querer ficar com o filho enquanto ele era atendido e um dos questionários ficou sem resposta a essa questão. No ano de 2011, todos os participantes disseram ficar tranquilos.

Dentre os motivos descritos para justificar o sentimento de tranquilidade apontado pela maioria dos acompanhantes, tanto em 2010 quanto em 2011, foram registrados: a confiança no trabalho dos profissionais e na qualidade dos serviços prestados, o fato de as crianças ficarem tranquilas e divertirem-se na sala de espera, o carinho e atenção demonstrada pelos profissionais em relação aos pacientes, o fato de saber que o filho está sendo bem tratado e que gosta do atendimento, pois quando é chamado ele vai sem nenhuma resistência, pelo fato de os profissionais buscarem a criança na sala de espera para ser atendido, cumprimentarem, serem atenciosos, conversarem e demonstrarem respeito por elas, sendo isso considerado fator muito importante.
Como já foi dito anteriormente, um dos objetivos do trabalho na sala de espera é desenvolver ações voltadas para saúde bucal, por meio de orientações e diálogos com a clientela atendida, possibilitando maior cuidado consigo mesmo e, por conseguinte, a prevenção das cáries e de doenças periodontais. Entendendo que todo trabalho de educação em saúde, para ser efetivado, requer a priori que haja interesse das pessoas para as quais é direcionado em envolverse com ele, investigou-se nesta pesquisa se os acompanhantes dos pacientes gostariam de obter informações sobre saúde e higiene bucal na sala de espera. De acordo com o questionário aplicado, $100 \%$ das pessoas que o responderam em 2010 e $88,9 \%$ em 2011 disseram que sim.

Com relação aos hábitos de higiene bucal dos acompanhantes, $72,72 \%$ no ano de 2010 e $72,22 \%$ em 2011 disseram utilizar fio dental diariamente durante a escovação. $\mathrm{O}$ uso de enxaguante bucal foi citado por 27, 27\% dos acompanhantes em 2010 e 16,66\% em 2011.

Ainda em relação aos hábitos de higiene bucal dos acompanhantes, 63,63\% disseram fazer a higienização três (03) vezes ao dia e em 2011 o equivalente a $50 \%$ deles citou realizá-la também com esta mesma frequência (tabela 3 ).

\begin{tabular}{lcc}
\hline Frequência & Ano & Ano \\
& 2010 & 2011 \\
\hline 03 vezes ao dia & 07 & 09 \\
04 vezes ao dia & 01 & 03 \\
03 a 04 vezes ao dia & 01 & 04 \\
05 vezes ao dia & $\mathbf{0 0}$ & 01 \\
Frequentemente & $\mathbf{0 1}$ & 00 \\
Sem resposta & $\mathbf{0 1}$ & 01 \\
Total & $\mathbf{1 1}$ & 18 \\
\hline
\end{tabular}

Tabela 3. Frequência diária da higienização bucal (acompanhantes).

Fonte: pesquisa direta

De acordo com os dados obtidos em relação aos hábitos de higiene bucal das crianças, a maioria realiza a escovação diária pelo menos três vezes ao dia, fazendo uso do fio dental. Em 2010, segundo informações dos acompanhantes, $81,81 \%$ das crianças utilizavam creme dental e fio dental durante a escovação, sendo que $45,45 \%$ o faziam três (03) vezes ao dia e $27,27 \%$ quatro (04) vezes ao dia. No ano de 2011 os dados mostram que 
$61,11 \%$ dos pacientes atendidos faziam uso do fio dental durante a higienização bucal, pelo menos duas (02) vezes ao dia (tabelas 4 e 5).

\begin{tabular}{lcc}
\hline \multicolumn{1}{c}{ Material } & Ano & Ano \\
& 2010 & 2011 \\
\hline Escova, creme e fio dental & 04 & 06 \\
Escova, creme, fio dental e & 00 & 01 \\
enxaguantebucal & & \\
Creme, fio dental e enxaguante bucal & 02 & 00 \\
Creme dental e fio dental & 03 & 03 \\
Escova e fio dental & 00 & 01 \\
Escova e creme dental & 01 & 03 \\
Creme dental infantil com sabor & 00 & 01 \\
Sem resposta & 01 & 02 \\
Não sabe & 00 & 01 \\
Total & 11 & 18 \\
\hline Tabela 4. Frequência do material & utilizado & durante a \\
higienização bucal (pacientes). & &
\end{tabular}

Fonte: pesquisa direta

\begin{tabular}{lcc}
\hline Frequência & Ano 2010 & Ano 2011 \\
\hline 02 vezes ao dia & 02 & 02 \\
03 vezes ao dia & 05 & 06 \\
04 vezes ao dia & 03 & 03 \\
02 a 03 vezes ao dia & 00 & 01 \\
03 a 04 vezes ao dia & 00 & 03 \\
Sem resposta & 01 & 02 \\
Não sabe & 00 & 01 \\
Total & 11 & 18 \\
\hline
\end{tabular}

Tabela 5. Frequência diária da higienização bucal (pacientes).

Fonte: pesquisa direta

Os dados aqui apresentados reforçam a importância de uma aproximação cada vez maior dos profissionais e estudantes da área de saúde em relação à comunidade para a qual presta serviços, neste caso, ligados às clínicas odontológicas de ensino. $\mathrm{O}$ diálogo e $\mathrm{o}$ desenvolvimento de pesquisas junto à clientela atendida e seus acompanhantes, sem dúvida alguma, contribui para o conhecimento de suas expectativas, hábitos e conceitos em saúde. Isso possibilita maior sintonia da aprendizagem de futuros profissionais e da ação daqueles que já atuam como tal, com seus reais interesses e necessidades.

Esse aspecto é ressaltado nas discussões apresentadas por Gonçalves e Verdi ${ }^{9}$ em relação ao atendimento em clínicas odontológicas de ensino. Para essas autoras, é imprescindível ter-se uma visão integral do paciente e isso implica um processo que envolve ações, assimilação de condutas e aquisição de hábitos de futuros profissionais permeados por um aprendizado para além de procedimentos técnicos, envolvendo reflexões éticas.

\section{Conelusão}

Ao longo deste trabalho verificou-se que as atividades realizadas a partir do projeto na sala de espera têm sido importantes aliadas em relação a um atendimento odontológico que vai ao encontro dos interesses da comunidade a qual serve, promovendo educação em saúde com vistas aos aspectos preventivos, bem como, um tratamento sensível às questões emocionais dos pacientes envolvidos, gerando bem-estar à medida que reduz a ansiedade característica que permeia a atuação clínica em odontologia.

Outro aspecto relevante das atividades desenvolvidas em sala de espera refere-se à formação dos estudantes do Curso Técnico em Saúde Bucal, uma vez que lhes possibilita experiências e aquisição de conhecimentos relativos aos processos de comunicação e relações interpessoais, tão importantes no exercício profissional $^{10}$.

Ao analisar os dados obtidos, devemos considerar a observação feita anteriormente em não se descartar a possibilidade de que os sujeitos tenham respondido às questões referentes à higienização bucal com base não em seu comportamento habitual e dos menores por eles acompanhados, mas em função das informações trabalhadas na sala de espera sobre a forma e a frequência adequadas de realizá-la. Entretanto, deve ser considerada também a possibilidade que este trabalho evoca em relação à mudança de comportamento frente à saúde bucal daqueles que dele participam.

Os dados obtidos revelam ainda a importância das informações e esclarecimentos prestados aos pais e/ou responsáveis pelos menores, ao término de cada sessão realizada durante o atendimento odontológico, uma vez que isso possibilita o desenvolvimento da confiança no trabalho do profissional bem como, pela influência desse conhecimento na melhoria das condições de higiene e saúde bucal das crianças, explicitado 
claramente pelos participantes desta pesquisa. Ao contrário, a falta de contato e envolvimento dos profissionais com os acompanhantes, além de deixá-los inseguros, gera desconfiança quanto à qualidade dos serviços prestados e não os estimula a um maior comprometimento com a saúde bucal dos menores em sua vida diária.

\section{Referências}

1. Paixão, NRDA, Castro, ARM. Grupo sala de espera: trabalho multiprofissional em unidade básica de saúde. Boletim da Saúde 2006; 20(2): 71-78, jul-dez.

2. Silva, DB, Detanico, MC, Bighetti, TI. Atuação da Odontologia no Projeto Sala de Espera da USB, Vila Municipal Pelotas, RS. In: Anais do XVIII Congresso de Iniciação Científica; XI Encontro de Pós-Graduação e I Mostra Científica da Universidade Federal de Pelotas; 2009; Pelotas. p. 20-23.

3. Pauleto, ARC, Pereira, MLT, Cyrino, EG. Saúde bucal: uma revisão crítica sobre programações educativas para escolares. Ciência e Saúde Coletiva 2004; 9(1): 121- 130. <Disponível em: http://www.scielo.org/php/index.php>. Acesso em: 10 jun. 2013.

4. Possobon, RF, Carrascoza, KC, Moraes, ABA; Costa JR, AL. O tratamento odontológico como gerador de ansiedade. Psicologia em Estudo 2007; 12(3): 609-616. $<$ Disponível em: http://www.scielo.org/php/index.php>. Acesso em: 14 ago. 2013.

5. Cardoso, CL, Loureiro, SR. Problemas comportamentais e stress em crianças com ansiedade frente ao tratamento odontológico. Estudos de Psicologia, 2005; 22 (1): 5- 12, jan-mar.

6. Cardoso, CL, Loureiro, SR. Estresse e comportamento de colaboração em face do tratamento odontopediátrico. Psicologia em Estudo 2008; 13(1): 133-141. Disponível em: <http://www.scielo.org/php/index.php>. Acesso em 16 out. 2012.

7. Tomita, NE, Pernambuco, RA, Lauris, JRP, Lopes, ES. Educação em saúde bucal para adolescentes: uso de métodos participativos. Revista da Faculdade de Odontologia de Bauru 2001; 9(1/2): 63-69. Disponível em:

$<$ http://sddinforma.files.wordpress.com/2010/07/2001 109.pdf $>$. Acesso em: 25 set. 2013.

8. Marra, SMP, Arciere, RM. Análise da inclusão da odontologia nos planos municipais de saúde das cidades integrantes da gerência regional de saúde de Uberlândia-MG. BioscienceJournal 2011; 27(3): 510-517, mai-jun.

9. Gonçalves, ER, Verdi, MIM. Os problemas éticos no atendimento a pacientes na clínica odontológica de ensino. Ciência e Saúde Coletiva 2007; 12(3): 755- 764. Disponível em: <http://www.scielo.org/php/index.php>. Acesso em: 18 nov. 2013.

10. Stutz, BL. Explorando o desenho para redução da ansiedade infantil na sala de espera em odontologia. Revista Em Extensão 2011; 10(2): 162- 171, jul-dez. 